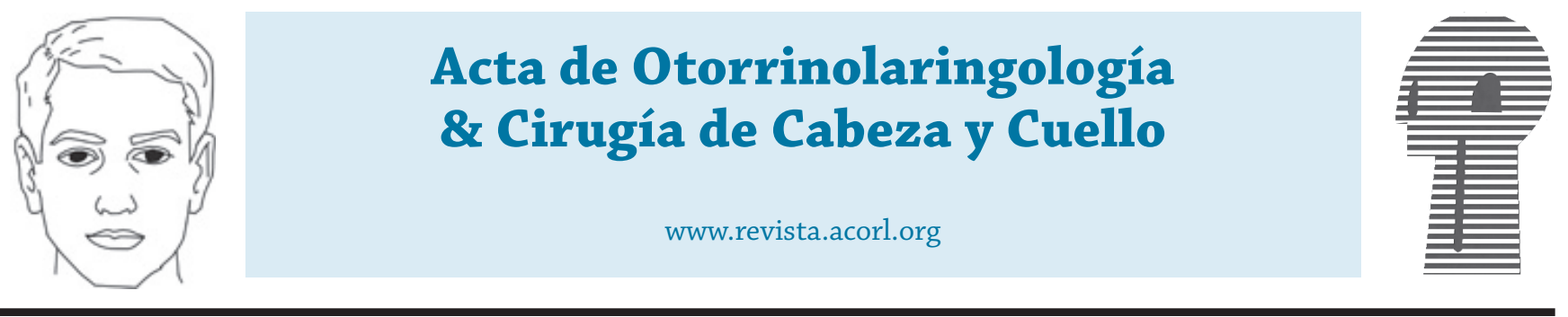

Trabajos originales

\title{
Ángulo de convexidad facial y proyección del mentón en pacientes en postoperatorio de rinoseptoplastia.
}

\section{Angle facial convexity and projection of the chin in patients undergone of rhinoplasty.}

\author{
Fernández - Fernández Karla Daniela*, López-Ulloa F**.
}

* Otorrinolaringóloga y cirujano de cabeza y cuello, alumna del diplomado de Rinología y Cirugía Facial de la Universidad Nacional Autónoma de México. Especialista en Unidad de Otorrinolaringología de la Clínica de Marly, Bogotá Colombia.

** Otorrinolaringólogo y cirujano de cabeza y cuello, especialista en Rinología y cirugía plástica facial, profesor adscrito al diplomado de Rinología y cirugía facial de la UNAM, Ciudad de México.

Forma de citar: Fernández - Fernández KD, López-Ulloa F. Ángulo de convexidad facial y proyección del mentón en pacientes en postoperatorio de rinoseptoplastia. Acta otorrinolaringol. cir. cabeza cuello. 2019;47(1):47-52. Doi: 10.37076/acorl.v47i1.293

\section{INFORMACIÓN DEL ARTÍCULO}

\section{Historia del artículo:}

Recibido: 28 de Agosto de 2018

Evaluado: 28 de Octubre de 2018

Aceptado: 28 de Diciembre de 2018

Palabras clave (DeCS):

Rinoseptoplastia, Mentón, Tamaño

Corporal.

\section{RESUMEN}

Introducción: La relación de la nariz con la morfología facial no es solo estructural. Numerosos estudios sugieren la relación entre la función respiratoria nasal y el desarrollo craneofacial. Objetivo: Determinar la mejoría en el ángulo de convexidad facial y proyección del mentón en pacientes en postoperatorio de rinoseptoplastia. Diseño: Estudio observacional analítico tipo corte transversal. Metodología: Muestra de 43 pacientes, 26 de género femenino y 17 masculino, entre 12 y 43 años, sometidos a rinoseptoplastia con evolución postoperatoria mínima de 3 meses. Se analizaron las variables de edad, sexo, ángulo de convexidad facial de Legan y proyección del mentón según González Ulloa antes y después de la cirugía, así como tiempo postquirúrgico. Resultados: El ángulo de convexidad facial se redujo $3,72^{\circ}+2,19$. En la técnica de González Ulloa, se evidenció avance del pogonion $2,53 \mathrm{~mm}+1,60$. De los 43 pacientes, un 72\%, mostró cambios favorables en el ángulo de convexidad facial y en la proyección del mentón. Los mejores resultados se evidenciaron en menores de 16 años y evolución postquirúrgica mayor a 1 año. Discusión: A diferencia de otras investigaciones, observamos cambios significativos 
Key words (MeSH):

Rhinoplasty, Chin, Body Size. en pacientes adultos. Tal hecho nos lleva a otras interrogantes sobre mecanismos adicionales que pudieran influir en los patrones de crecimiento facial. Conclusiones: La rinoseptoplastia al considerarse un procedimiento que mejora la función respiratoria nasal, favorece cambios neuromusculares y endocrinos que permiten un mejor desarrollo del perfil facial en especial del tercio inferior.

\section{ABSTRACT}

Introduction: The relation between the nose and the facial morphology is not only structural. Numerous studies have demonstrated also a relationship between nasal respiratory function and craniofacial development. Objective: To determine the improvement in the angle of facial convexity and chin projection in patients in the postoperative period of rhinoseptoplasty. Design: Cross-sectional study. Methods: 43 patients were selected. 26 women and 17 men between 12 and 43 years old, undergoing Rhinoplasty with minimum 3 months of postoperative evolution. We analyzed the variables of age, sex, Legan's angle of facial convexity and projection of the chin according to Gonzalez Ulloa before and after surgery, as well as postsurgical time. Results: The angle of facial convexity was reduced X + D.S 3.72 $\circ+2.19$. The technique of González Ulloa showed progress of the pogonion $\mathrm{X}+$ D.S $2.53 \mathrm{~mm}+1.60$. Of the 43 patients, $72 \%$, showed positive changes at the angle of facial convexity and the projection of the Chin. Best results were apparent in children under 16 years and more than 1 year postsurgical evolution. Discussion: Unlike other research, we observe significant changes in adult patients. This fact leads to other questions about additional mechanisms that might influence the facial growth patterns. Conclusions: Rhinoseptoplasty is a procedure that improves the nasal respiratory function, favors neuromuscular and endocrine changes that allow better development of the facial profile especially in the lower third.

\section{Introducción}

Como parte del análisis estético y funcional de las proporciones faciales, se incluye la nariz como un todo y no como un componente predominante. El cirujano estudioso de este tema está educado para la observación meticulosa de dichas proporciones. Otras estructuras faciales también tienen gran influencia estética, como aquellas que se encuentran en el tercio inferior como lo es el mentón, el cual puede ser definido en cuanto a posición y tamaño ya que influye en la armonía facial (1).

Sin embargo, la relación de la nariz con la morfología facial no es solo desde el punto de vista estructural. Por el contrario, numerosos estudios clínicos, han sugerido una estrecha relación entre la función respiratoria nasal y su impacto en el desarrollo craneofacial. Todo ello basado en la fisiología respiratoria, según la cual el aire ingresa libremente por la nariz con un cierre inmediato de la boca, elevación y proyección de la lengua contra el paladar, ejerciendo un estímulo positivo para su desarrollo. En presencia de algún factor que obstruya la vía aérea superior como hipertrofia de adenoides, rinitis alérgica, desviación septal y deformidades de la pirámide nasal, se activa la respiración bucal, en la cual la mandíbula desciende, la lengua se ubica en el piso de la boca, disminuyendo la presión sobre el maxilar superior (2).
En casos crónicos, el mantenimiento de la respiración bucal origina retracción y descenso mandibular, elongación vertical del tercio facial inferior, ángulo mandibular obtuso, paladar estrecho y elevado, alargamiento de la cara, maloclusión y mordida cruzada con consecuencias adversas en la masticación y la fonación, así como en la estética facial, cambios con mayor influencia en la fase rápida de crecimiento entre los 3 y 5 años de edad (3).

La mayoría de las investigaciones se han basado en realizar análisis cefalométricos en pacientes pediátricos con hipertrofia adenotonsilar, describiendo el típico patrón de respirador bucal. Kerr y colaboradores, analizaron el patrón de crecimiento mandibular en 26 niños tratados por obstrucción nasal mediante adenoidectomía, mostrando cambios en la respiración posterior a la cirugía y con ello reversibilidad de las alteraciones en el crecimiento mandibular (1). Por otra parte, es bien sabido que la pirámide nasal juega un papel fundamental en la fisiología respiratoria. Las deformidades estéticas bien sea congénitas o adquiridas, pueden tener un impacto en la insuficiencia respiratoria nasal y con ello repercutir en el desarrollo craneofacial, así como en el aspecto estético de los tejidos blandos (4).

Dentro de las alteraciones del desarrollo facial, la que mayormente se analiza en relación a la rinoplastia por su influencia directa en los resultados es la microgenia o retrognatia, siendo necesario muchas veces colocar implantes 
de mentón o realizar genioplastia para conseguir el balance facial estéticamente aceptable (5).

Por lo tanto, si se toma en cuenta la fisiología nasal y los cambios musculoesqueléticos que ocurren al revertir el patrón respiratorio, sería interesante evaluar la evolución de los pacientes sometidos a rinoseptoplastia en relación al ángulo de convexidad facial y proyección del mentón como métodos utilizados para evaluar el perfil facial.

Por tal motivo, el objetivo de la presente investigación es determinar la mejoría en el ángulo de convexidad facial y proyección del mentón según la técnica de González Ulloa en pacientes en postoperatorio de rinoseptoplastia integral sin mentoplastia.

\section{Metodología}

Se realizó una investigación observacional analítica tipo corte transversal. Se revisó los registros de historias clínicas en la consulta de Rinología de la Clínica Zurich en Ciudad de México entre el período marzo-mayo de 2018. Se obtuvo una muestra intencional, no probabilística, seleccionando 43 pacientes de ambos sexos con deformidad de la pirámide nasal, rinoseptodesviación y obstrucción nasal con retrognatia asociada, sometidos a rinoseptoplastia entre los 12 y 39 años de edad.

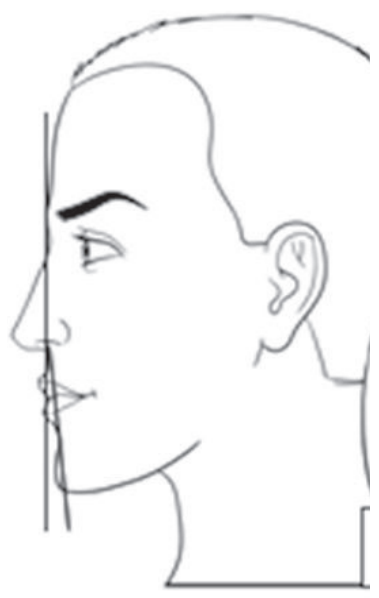

Figura 1. Ángulo de convexidad facial de Legan. Se traza una línea glabela-subnasal, interceptada por una línea subnasal- pogonión. El ángulo de convexidad debe ser de 12 grados.

Se analizaron fotografías de perfil pre y postquirúrgicas a partir de 3 meses, tomadas con cámara profesional (Sony, Japón) fondo azul a 1.30 metros del paciente. Con el plano de Frankfurt paralelo al piso, labios en reposo, cabello detrás de las orejas, sin anteojos. Se realizó medición numérica en grados de los ángulos de convexidad facial de Legan (ACF), (figura 1) y de la proyección del mentón (PM) en milímetros mediante la técnica de González Ulloa (Gu), (figura 2).

Se compararon los resultados pre y postoperatorios, tomando como base de normalidad $12^{\circ}$ para el ángulo de

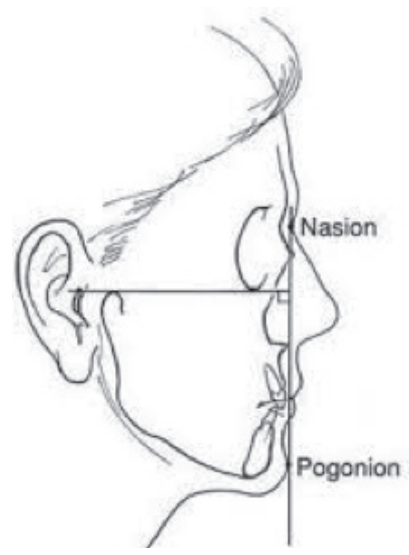

Figura 2. Método González- Ulloa de proyección del mentón. Línea perpendicular al plano de Frankfurt a nivel del nasion. El pogonion debe estar a nivel del meridiano 0.

convexidad facial, y pogonion a nivel del meridiano 0 en el método González Ulloa (figura 3 y 4).

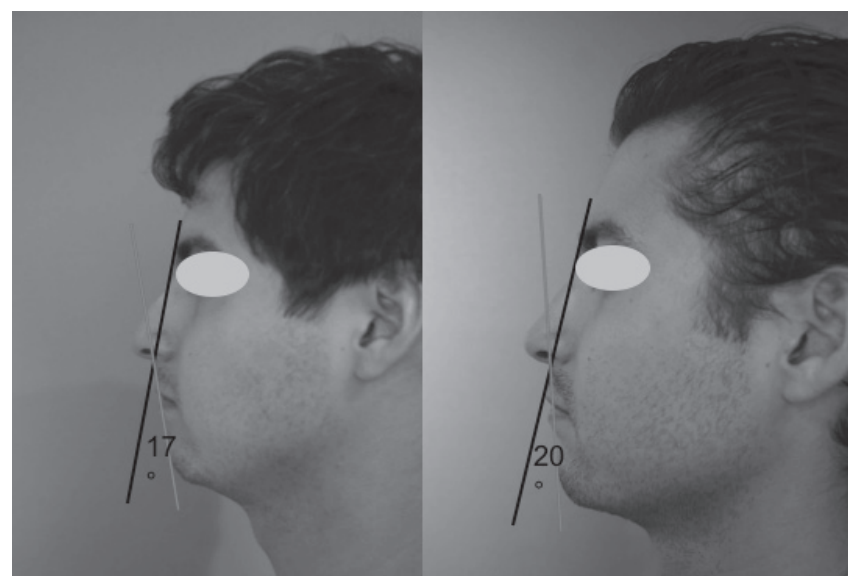

Figura 3. Medición del ángulo de convexidad facial. En este paciente varía de $20^{\circ}$ en el preoperatorio a $17^{\circ}$ en el postoperatorio (normal $12^{\circ}$ ).

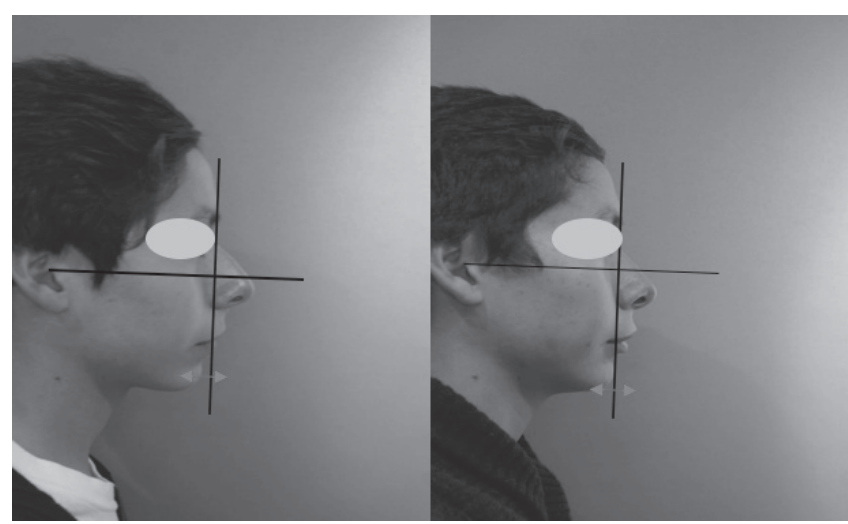

Figura 4. Medición del ángulo de convexidad facial. En este paciente varía de $20^{\circ}$ en el preoperatorio a $17^{\circ}$ en el postoperatorio (normal $12^{\circ}$ ). 


\section{Análisis estadístico}

Se efectuó un análisis de los datos mediante medias y desviación estándar por grupo etario y género. Asimismo, se realizó un análisis inferencial comparando mediciones pre y postquirúrgicas y su razón de mejoría, considerando significativo una diferencia menor a 0,05 mediante la prueba de t-Student, con intervalo de confianza de $95 \%$

\section{Aspectos éticos}

De acuerdo a la Ley General de Salud, se considera esta investigación como CATEGORIA I, con riesgo menor al mínimo. La información recabada sólo se utilizó para cumplir con los objetivos de la investigación, además de ser confidencial y por tal razón se omitirá los nombres propios. Se obtuvo autorización de los médicos tratantes para el acceso al expediente clínico y fotográfico de los participantes a modo de recabar información complementaria necesaria en el seguimiento de los participantes.

\section{Resultados}

Del total de 43 pacientes evaluados, el rango de edad estuvo entre 12 y 39 años con una media de 18,9 años D.S + 5, de los cuales 17 fueron masculinos (39\%) y 26 femeninos (60\%).

Según el rango de edad fueron divididos en 2 grupos: aquellos mayores de 16 años (33 pacientes, 76\%) y el grupo menor de 16 años (10 pacientes, 23\%). Del primer grupo 22 femeninos (66\%) y 11 masculinos (33\%). Del segundo grupo 4 femeninos (40\%) mientras que $6(60 \%)$ son masculinos.

Del total de los pacientes, un 72\% (31 pacientes) mostró cambios favorables tanto en el ángulo de convexidad facial (ACF) como en la proyección del mentón (PM) según GU, mientras que en $28 \%$ (12) no se consiguió ninguna modificación.

El ángulo de convexidad facial prequirúrgico presentó $\mathrm{X}+\mathrm{D} . \mathrm{S} 18,14^{\circ}+3,43$ y de valor postquirúrgico $\mathrm{X}+$ D.S $14,2^{\circ}+2,97$, con una mejoría de X + D.S $3,72^{\circ}+2.19$, $\mathrm{p}<0.05$, lo cual es estadísticamente significativo. La proyección del mentón según GU presentó en el preoperatorio $\mathrm{X}+$ D.S $4,49 \mathrm{~mm}+1,82 \mathrm{y}$ en el postoperatorio $\mathrm{X}+\mathrm{D} . \mathrm{S}$ $1,95 \mathrm{~mm}+1,06$, con mejoría de X + D.S 2,53 mm + 1,60, igualmente significativo, $\mathrm{p}<0.05$ con intervalo de confianza superior a $95 \%$. (Tabla 1 )

Tabla 1. Ángulo de convexidad facial y proyección del mentón pre y postquirúrgico.

\begin{tabular}{|l|c|c|c|c|}
\hline \multicolumn{1}{|c|}{$\mathrm{n}=43$} & $\begin{array}{c}\text { Pre } \\
\text { quirúrgico }\end{array}$ & $\begin{array}{c}\text { Post } \\
\text { quirúrgico }\end{array}$ & Mejoría & $\mathrm{p}$ \\
\hline $\begin{array}{l}\text { Angulo de } \\
\text { convexidad } \\
\text { facial } \\
\text { (grados) }\end{array}$ & $18,14+3,43$ & $14,42+2,97$ & $3,72+2,19$ & $\mathrm{p}<0.05$ \\
\hline $\begin{array}{l}\text { Proyección } \\
\text { del mentón } \\
\text { (milímetros) }\end{array}$ & $4,49+1,82$ & $1,95+1,06$ & $2,53+1,60$ & $\mathrm{p}<0.05$ \\
\hline
\end{tabular}

En relación al tiempo postoperatorio, se observó una mayor variación en el grupo mayor a un año de evolución presentando mejoría del ACF X + D.S 3,62 ${ }^{\circ}+2.19$ y en la PM X + D.S 2,25 mm + 1,32. En contraste con el grupo de 3 a 12 meses de evolución postquirúrgica que presentó variación en ACF X + D.S 2,55 + 1,51 y en PM X + D.S $1,88 \mathrm{~mm}+1,11$. Dichos resultados fueron estadísticamente significativos, $\mathrm{p}=0,03$ y 0.04 respectivamente (figura 5).

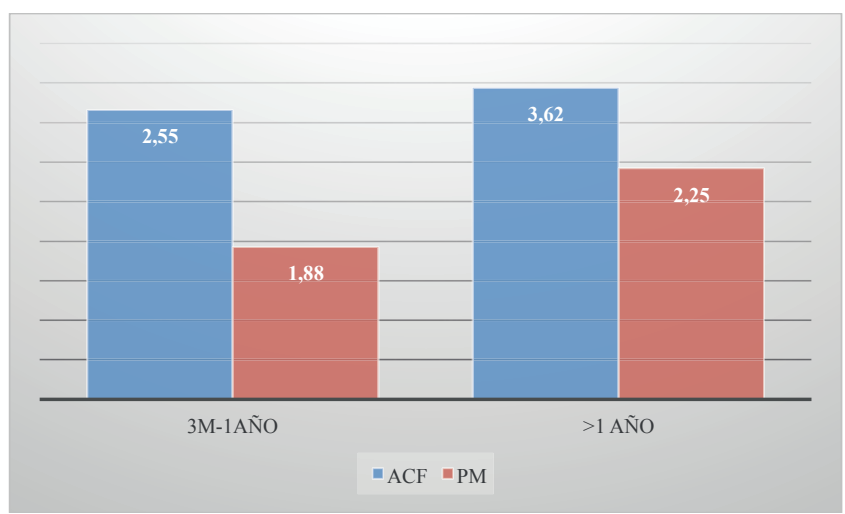

Figura 5. MMedia de mejoría según el tiempo postquirúrgico del ángulo de convexidad facial (ACF) en grados y proyección del mentón según González Ulloa (PM) en milímetros.

El género masculino presentó mejoría discretamente superior en el ángulo de convexidad facial con respecto al género femenino en ambos grupos de edades. Sin embargo, esto no fue estadísticamente significativo, $p>0,05$. En relación a la proyección del mentón se observó una mejoría muy superior en los varones menores de 16 años con respecto a las mujeres, $\mathrm{p}=0.002$.

En cuanto a los cambios según edad, se pudieron observar que el grupo menor a 16 años presentó mayores modificaciones en ambas mediciones respecto al grupo mayor de 16 años, sin embargo, no fue altamente significativo $\mathrm{p}>0,05$ (Tabla 2).

Tabla 2. Mejoría del ángulo de convexidad facial y proyección del mentón según grupo de edad X + D.S

\begin{tabular}{|l|c|c|c|}
\hline \multicolumn{1}{|c|}{ Parámetro } & $\begin{array}{c}\text { Menores } 16 \\
\mathrm{n}=10\end{array}$ & $\begin{array}{c}\text { Mayores } 16 \\
\mathrm{n}=33\end{array}$ & $\mathrm{p}$ \\
\hline $\begin{array}{l}\text { Angulo de convexidad } \\
\text { facial (grados) }\end{array}$ & $4,62+3,15$ & $3,51+1,84$ & 0,2 * \\
\hline $\begin{array}{l}\text { Proyección del mentón } \\
\text { (milímetros) }\end{array}$ & $3,25+0,96$ & $2,37+1,67$ & 0,1 * \\
\hline
\end{tabular}

\section{Discusión}

Son numerosos los estudios que han explicado la relación entre la respiración nasal y su efecto en el desarrollo craneofacial. La mayoría de dichas investigaciones se han basado en realizar análisis cefalométricos en pacientes pediátricos con hipertrofia adenotonsilar, describiendo el típico patrón de respirador bucal o la conocida fascies adenoidea caracterizada principalmente por incompetencia del labio inferior, 
paladar superior alto y estrecho, tercio inferior alargado, ángulo gonial obtuso y retrognatia. Linder Aronso en 1970 fue el primer autor en describir dicha relación (6).

Posteriormente, Kerr y colaboradores en 1989 extendieron el estudio de Linder, observando los cambios mandibulares en los mismos pacientes sometidos a adenoidectomía, obteniendo resultados interesantes con reversión de las anormalidades cefalométricas en la mayoría de los pacientes (2).

En 1999, Bar y colaboradores reportan un incremento en la secreción de Hormona del Crecimiento y Factor de Crecimiento parecido a la Insulina en pacientes operados de adenotonsilectomía, lo cual pudiera explicar los cambios experimentados en cuanto a desarrollo craneofacial en niños, asociado a los cambios neuromusculares favorecidos por la respiración nasal y el cierre bucal. Sin embargo, poco se ha investigado sobre el comportamiento de estos parámetros a nivel de tejidos blandos en pacientes con trastornos de vía aérea superior diferente a la hipertrofia adenotonsilar y en pacientes mayores de 16 años, en los cuales ya el crecimiento óseo habría culminado (7).

En la presente investigación se analizó el perfil facial de pacientes menores y mayores a 17 años de edad con obstrucción nasal y deformidad de la pirámide nasal en búsqueda de cambios en el perfil facial, específicamente en cuanto a ángulo de convexidad facial y proyección del mentón, así como los cambios experimentados a partir de los 3 meses de realizada la rinoplastia, consiguiendo resultados interesantes.

Al igual que en las investigaciones previamente comentadas, los pacientes de nuestro estudio presentaron alteraciones del perfil facial especialmente retrognatia asociado a respiración bucal por disfunción respiratoria nasal secundaria a trastornos de la pirámide nasal. Esto coincide con los reportes de la literatura en cuanto a la relación vía aérea superior- patrón craneofacial y ortognático.

Según nuestros resultados, a partir de los 3 meses de la cirugía, se exhibieron cambios favorables en cuanto a proyección del mentón medido con el ángulo de convexidad de Legan, observándose reducción significativa del mismo, así como proyección del mentón según la técnica de González Ulloa, siendo estos los cambios más marcados a partir del primer año de postoperatorio. Este hecho contrasta con los resultados de Woodside en 1991, en cuyos pacientes no hubo cambios hasta 5 años posterior a la adenoidectomía (8).

En cuanto a la comparación por género, no existieron diferencias significativas entre masculinos y femeninos; sin embargo, fue discretamente superior en los masculinos, esto podría explicarse por la influencia de la testosterona y su repercusión en el crecimiento.

En nuestro estudio, el grupo de pacientes con mayor razón de mejoría fue el grupo menor a 16 años, lo cual se justifica por la mayor tasa de crecimiento óseo en esas edades y pudiera asimismo explicarse por la teoría del incremento de la Hormona de Crecimiento al restaurarse la respiración nasal como lo describe Arlan L. Rosenbloom en su estudio sobre fisiología del crecimiento (7).
Por otra parte, es importante destacar el cambio experimentado por los pacientes mayores de 16 años de edad, quienes presentaron un desarrollo significativo de la proyección del mentón a pesar de haber completado la pubertad y por tanto la fase de crecimiento óseo, resultados que no mostraron diferencia estadísticamente significativa con los del grupo de menor edad. Tal hecho nos lleva a otros interrogantes sobre mecanismos adicionales que pudieran influir en los patrones de crecimiento facial en pacientes adultos; o si los cambios neuromusculares favorecidos por la respiración nasal son lo suficientemente fuertes y constantes en el tiempo para modificar una morfología ya constituida.

\section{Conclusión}

La presente tuvo como objetivo principal determinar la mejoría en el ángulo de convexidad facial y proyección del mentón en pacientes operados de rinoseptoplastia. Los resultados obtenidos apoyan y ratifican la relación entre la función respiratoria nasal y los patrones de crecimiento craneofacial; asimismo sugieren que la rinoseptoplastia al considerarse un procedimiento que mejora la función respiratoria nasal, puede favorecer cambios neuromusculares y endocrinos permitiendo así un mejor desarrollo del perfil facial a mediano y largo plazo, y armonizando espontáneamente la estética facial, en muchos casos sin necesidad de mentoplastia o genioplastia de avance. Comportamiento que es posible observar incluso en buena proporción de pacientes adultos, siendo necesarias otras investigaciones con el fin de determinar otros factores involucrados en dichos cambios.

\section{Conflicto de interés}

Los autores abajo firmantes declaran no tener asociación comercial que pueda generar conflictos de interés en relación con el manuscrito.

\section{Financiación}

Las fuentes de financiación del trabajo presentado en este artículo están representadas por recursos económicos propios de los autores.

\section{Agradecimientos}

A todos los profesores del Diplomado de Rinología y Cirugía Facial del Hospital Ángeles de las Lomas, en especial al Dr. Fausto López Ulloa y la Dra. Irenne Maulen. A la División de Postgrado de Medicina de la UNAM.

\section{REFERENCIAS}

1. Chang EW, Meyers AD. Nasal Anatomy [Internet]. Nueva York: Medscape; 2015 Jul 23 [Citado 2018 Feb 1]. Disponible en: https://emedicine.medscape.com/article/835134-overview

2. Principato JJ. Upper airway obstruction and craniofaciaI 
morphology. Otolaryngol Head Neck Surg. 1991;104(6):881-90.

3. Belmont-Laguna F, Godina-Hernández G, Ceballos-Hernández H. El papel del pediatra ante el síndrome de respiración buccal. Acta Pediatr Mex. 2008;29(1):3-8.

4. Sociedad Española de Otorrinolaringología y Cirugía de Cabeza y Cuello. Libro virtual de formación en Otorrinolaringología. [Internet]. Madrid: SEORL PCF; 2007. Capítulo 47 Patología de la pirámide nasal y del vestíbulo. malformaciones de la nariz y de los senos paranasales. [Citado 2018 Jun 11]. Disponible en: https://seorl.net/

5. Capítulo 144 Procedimientos auxiliares a la rinoplastia: mentoplastia. En: Suarez- nieto, C. Tratado de Otorrinolaringología y Cirugía de Cabeza y Cuello. $2^{\mathrm{a}}$ Ed. Panamericana: Madrid, España: 2015.

6. García-Molina GI. Etiología y Diagnóstico de pacientes Respiradores Bucales en edades tempranas. Revista Latinoamericana de Ortodoncia y Odontopediatría. 2011.

7. Rosenbloom AL. Fisiología del crecimiento, Ann Nestlé [Esp]. 2007; 65:99-110.

8. Carbone DN. Análisis de los ángulos de convexidad facial y nasolabial en fotografías de niños respecto a sus distintos estadios de dentición en oclusión normal y maloclusiones. [Tesis de especialidad]. [Perú]: Universidad Peruana Cayetano Heredia; 2011. 56 p.

9. Puigdoger A. Análisis estético y funcional de tejidos blandos. Rev Esp Ortod. 2000;30:182-188.

10. Arroyo HH, Olivetti IP, Lima LF, Jurado JR. Clinical evaluation for chin augmentation: literature review and algorithm proposal. Braz J Otorhinolaryngol 2016;82(5):596-601.

11. Peltomäki T. The effect of mode of breathing on craniofacial growth revisited. Eur J Orthod. 2007;29(5):426-9.

12. Frodel JL, Sykes JM, Jones JL. Evaluation and treatment of vertical microgenia. Arch Facial Plast Surg. 2004;6(2):111-119

13. Ahmed J, Patil S, Jayaraj S. Assessment of the chin in patients undergoing rhinoplasty: what proportion may benefit from chin augmentation? Otolaryngol Head Neck Surg. 142(2):164-8. 